\title{
Contribuições ao conhecimento do gênero Paraceresa Kopp \& Yonke (Hemiptera, Auchenorrhyncha, Membracidae)
}

\author{
Gabriel Simões de Andrade
}

Centro de Ciências Biológicas e da Saúde, Universidade Estadual do Oeste do Paraná. Rua Universitária 2069, Caixa Postal 711, 85819-110 Cascavel, Paraná, Brasil.E-mail: gsa@certto.com.br

\begin{abstract}
Contributions to the knowledge of the genus Paraceresa Kopp \& Yonke (Hemiptera, Auchenorrhyncha, Membracidae). A recharacterization of the genus Paraceresa Kopp \& Yonke, 1979, the new combinations P. chacoensis (Remes-Lenicov, 1973), P. colon (Germar, 1835), with Ceresa recta Walker, 1858 as a new synonym, P. ornithisca (Remes-Lenicov, 1978) and P. spinosa (Remes-Lenicov, 1973), and a check list of recognized species are presented.

KEY WORDS. Ceresini, Membracoidea, Smiliinae, taxonomy.
\end{abstract}

RESUMO. São apresentadas a recaracterização do gênero Paraceresa Kopp \& Yonke, 1979, as novas combinações $P$. chacoensis (Remes-Lenicov, 1973), P. colon (Germar, 1835), esta com Ceresa recta Walker, 1858 como novo sinônimo, $P$. ornithisca (Remes-Lenicov, 1978) e P. spinosa (Remes-Lenicov, 1973), bem como lista remissiva das espécies reconhecidas. PALAVRAS CHAVE. Ceresini, Membracoidea, Smiliinae, taxonomia.

Paraceresa Kopp \& Yonke, 1979 foi descrito para acomodar sete espécies neotropicais, cinco das quais descritas por ReMEs-LenICOV (1970, 1971, 1973a) em gêneros cujas espécies são de distribuição neártica. Durante estudos visando a análise cladística dos Ceresini foram levantados novos caracteres e detectadas mais espécies que se classificam no gênero, o que é apresentado a seguir como subsídios para futura revisão dos táxons.

As siglas utilizadas para referenciar o repositório dos tipos e o nome das instituições por extenso são: (BMNH) The Natural History Museum, Londres, Inglaterra; (DZUP) Departamento de Zoologia, Universidade Federal do Paraná, Curitiba, Brasil; (MLPA) Facultad de Ciencias Naturales y Museo, La Plata, Argentina; (MRSN) Museo Regionale di Scienze Naturali, Torino, Itália; (NHRS) Naturhistoriska Riksmuseet, Estocolmo, Suécia.

\section{Paraceresa Kopp \& Yonke, 1979}

Paraceresa Kopp \& Yonke, 1979: 28. Espécie-tipo: Ceresa bifasciata Fairmaire, 1846, por designação original.

Descrição. Tamanho pequeno a médio, variando entre 5,0 e 8,0 milímetros. Coloração amarelada, usualmente com bandas escurecidas no pronoto posterior. Cabeça com a superfície esculturada em circunvoluções. Pronoto acuminado, com o metopídio distintamente diferenciado das regiões supra-umerais em vista frontal; processos supra-umerais presentes, usualmente desenvolvidos e cônicos; sulco supra-umerais distintos; regiões supra-umerais expandidas; processo pronotal posterior subulado ao menos no terço apical; carena dorsal arredondada.
Genitália masculina. Placas laterais do pigóforo com a margem superior largamente arredondada; denticulação mediana curta e robusta. Placa subgenital triangular, incisão apical variável. Parâmeros bem desenvolvidos, metade distal fortemente curvada, ápice variadamente modificado. Edeágo em forma de "U" em vista lateral, armação anterior eventualmente reduzida, armação posterior tubular na base, gradualmente comprimida ântero-posteriormente e afilada em direção ao ápice; orifício funcional alongado.

Espécies incluídas:

Paraceresa bifasciata (Fairmaire, 1846: 286) (Ceresa). Síntipo fêmea (MRSN; examinado). Localidade-tipo: "Brésil...".

Paraceresa biguttata (Remes-Lenicov, 1971: 126, lám. I, figs 1-7) (Anisostylus). Holótipo macho (DZUP). Localidade-tipo: BRASIL, Paraná: Três Barras.

Paraceresa brasiliensis (Remes-Lenicov, 1971: 127, lám. II, figs 8-16) (Anisostylus). Holótipo macho (DZUP). Localidadetipo: Brasil, São Paulo: Serra da Bocaina.

Paraceresa chacoensis (Remes-Lenicov, 1973b: 53, lám. II, figs 10-15) (Spissistilus). Holótipo macho (MLPA). Localidadetipo: Argentina, Misiones: San Ignacio, comb. nov.

Paraceresa colon (Germar, 1835: 237) (Smilia). Localidade-tipo: "Habitat in Brasilia. Bescke.", comb. nov.

Ceresa recta Walker, 1858: 68. Lectótipo fêmea (BMNH; examinado). Localidade-tipo: "South America?", syn. nov. Paraceresa deltae (Remes-Lenicov, 1973a: 82, lám. I, figs 1-7) (Stictocephala). Holótipo macho (MLPA). Localidade-tipo: Argentina, Buenos Aires: San Fernando.

Revista Brasileira de Zoologia 21 (1): 39-41, março 2004 

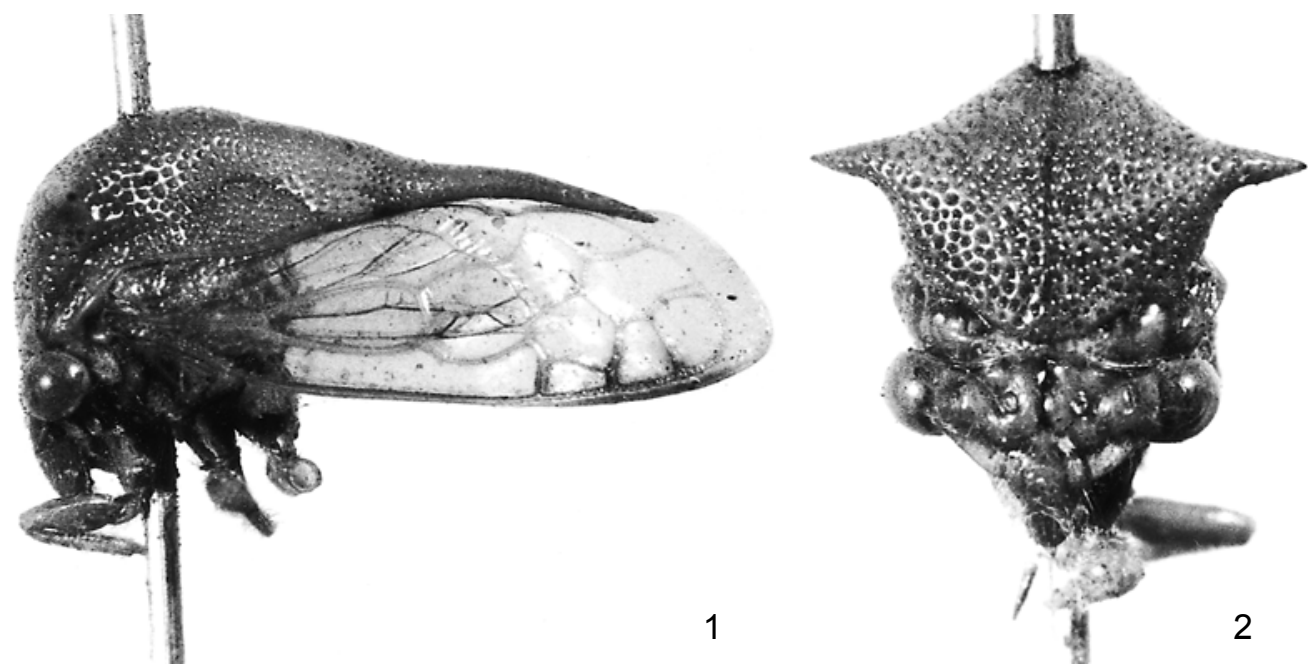

Figuras 1-2. Paraceresa colon: (1) vista lateral esquerda; (2) vista frontal.

Paraceresa ornithisca (Remes-Lenicov, 1978: 111, lám. I, figs 19) (Stictocephala). Holótipo macho (MLPA). Localidade-tipo: Brasil, Bahia: Senhor do Bonfim, comb. nov.

Paraceresa pallidinervis (Remes-Lenicov, 1970: 121, figs 12-22) (Stictocephala). Holótipo macho (MLPA). Localidade-tipo: Argentina, Salta: Rosario de la Frontera.

Paraceresa spinosa (Remes-Lenicov, 1973b: 51, lám. I, figs 1-9) (Spissistilus). Holótipo macho (MLPA). Localidade-tipo: ARGentina, Salta: Orán. comb. nov.

Paraceresa trifasciata (Remes-Lenicov, 1971: 128, lám. III, figs 17-25) (Anisostylus). Holótipo macho (MLPA). Localidadetipo: Argentina, Chaco: Laguna Blanca.

Paraceresa unguicularis (Stål, 1862: 26) (Ceresa). Holótipo macho (NHRS). Localidade-tipo: Brasil, Rio de Janeiro: Rio de Janeiro.

Stictocephala punctata Remes-Lenicov, 1973a: 84, lám. I, figs 8-16. Holótipo macho (MLPA). Localidade-tipo: ArgENTINA, Misiones: Loreto. Sinonimizada por Kopp \& YonKe (1979).

Comentários. Fairmaire (1846) incluiu C. bifasciata em sua primeira seção do gênero Ceresa, caracterizada como "I. Carène dorsale tranchante, épaules armées.", e ao tratar da espécie cita "Dans cette espèce la carène dorsale commence à s'arrondir.". Esta observação já era uma alusão aos caracteres da morfologia externa que constituem sinapomorfias de Paraceresa e que indicam parentesco com os gêneros de Ceresini ornamentados.

Este mesmo autor tratou de Smilia colon Germar, 1835 em sua segunda divisão do gênero Ceresa, caracterizada como "II. Carène dorsale arrondie, quelquefois noduleuse.", citando a procedência do material examinado como "Coll. Germar, Spinola.". O exame de um dos três exemplares existentes na coleção M. Spinola (MRSN) mostrou que o mesmo apresenta caracteres compatíveis com a descrição original e com a redescrição de Fairmaire (1846), e que pode ser realmente conspecífico com o material de E.F. Germar manipulado por Fairmaire. Segundo CASALE (1981), os dados constantes na etiqueta de caixa pertinente aos exemplares são "Ceresa colon Germar, Brésil e Buquet". Além dos aspectos do pronoto acima referidos, as estruturas da genitália do exemplar, macho, mostram que esta espécie classifica-se em Paraceresa.

O estudo comparativo do lectótipo de Ceresa recta Walker, 1858 com o material de $P$. colon mostrou que se tratam da mesma espécie.

As alterações nomenclaturais de Spissistilus chacoensis Remes-Lenicov, Spissistilus spinosus Remes-Lenicov e de Stictocephala ornithisca Remes-Lenicov foram determinadas por estudo das descrições e ilustrações da autora, tendo em mente sobretudo que estes táxons em nível genérico são de ocorrência na América do Norte.

São sinapomorfias as configurações das placas laterais do pigóforo do macho em conjunto com as expressões do edeago e aspectos do pronoto.

\section{AGRADECIMENTOS}

Pelo empréstimo de material tipo a A. Casale (Museo Regionale di Scienze Naturali, Torino, Itália) e ao Prof. Dr. Albino Morimasa Sakakibara (Universidade Federal do Paraná, Curitiba, Brasil), pela confecção dos originais das fotografias.

\section{REFERÊNCIAS BIBLIOGRÁFICAS}

Casale, A. 1981. Cataloghi II - Collezione Emitterologica di Massimiliano Spinola. Torino, Museo Regionale di Scienze Naturali, 120p. 
Fairmaire, L.M.H. 1846. Revue de la tribu des Membracides. Annales de la Société Entomologique de France, Paris, 4: 235-320.

Germar, E.F. 1835. Species Membracidum Musae E.F. Germari. Revue Entomologique, Strasbourg, 3: 223-262.

Kopp, D.D. \& T.R. Yonke. 1979. A taxonomic review of the tribe Ceresini (Homoptera: Membracidae). Miscellaneous Publications of the Entomological Society of America, College Park, 11 (2): 1-97.

Remes-Lenicov, A.M.M. 1970. Notas sobre Membrácidos neotropicales. I. Stictocephala Stal (sic), nuevo género de Membrácido para la República Argentina (Homoptera). Revista de la Sociedad Entomológica Argentina, Buenos Aires, 32 (14): $117-122$.

- 1971. Notas sobre Membrácidos neotropicales. III. Tres especies nuevas de Anisostylus Caldwell (Homoptera). Revista de la Sociedad Entomológica Argentina, Buenos
Aires, 33 (1-4): 125-131.

. 1973a. Nota sobre Membrácidos neotropicales V (Insecta-Membracidae). Neotropica, La Plata, 19 (59): 8286.

1973b. Notas sobre Membrácidos neotropicales. IV. Dos especies nuevas de Spissistilus Caldwell (HomopteraMembracidae Revista de la Sociedad Entomológica Argentina, Buenos Aires, 34 (1-2): 51-55.

. 1978. Contribución al estudio de los Membrácidos neotropicales III (Insecta-Homoptera). Neotropica, La Plata, 24 (72): 111-116.

STÅL, C. 1862. Bidrag till Rio de Janeiro-traktens HemipterFauna. II. Öfversigt af Kongliga Svenska VetenskapsAkademiens Förhandlingar, Estocolmo, 3:1-75.

WALKER, F. 1858. Insecta saundersiana: or characteres of undescribed insects in the collection of William Wilson Saunders, Esq. Homoptera. London, J.V. Voorst, 117p.

Recebido em 02.VII.2003; aceito em 18.I.2004. 